\title{
A Uniformly Distributed Congruence
}

\author{
Darrell Cox and Sourangshu Ghosh
}

January 18, 2021

\begin{abstract}
An elementary congruence appears to be uniformly distributed. The Weyl criterion is used to confirm this.
\end{abstract}

\section{Introduction}

The congruence is $d(n-x)-(l-d) x \equiv-1 \bmod l$ where $l$ is a prime, $n$ is a natural number less than $l$, and $d$ is a natural number less than $l$ that satisfies the congruence $l \mid d n+1$. For $d=1,2,3, \ldots, l-1$, the solutions of $l \mid d n+1$ are a permutation of the natural numbers $(1,2,3, \ldots, l-1)$. When a solution is found, the $d$ and $n$ values are used to solve the first congruence. This is used by Cox et al.[1][2] to derive optimal bounds for the length of rational Collatz and $3 \mathrm{n}+\mathrm{c}$ cycles.

\section{$2(n-x, x)$ Values and Uniform Distributions}

For a real number $x$, let $[x]$ denote the integral part of $x$ and $\{x\}$ the fractional part. The sequence $\omega=\left\{x_{n}\right\}, n=1,2,3, \ldots$ of real numbers is said to be uniformly distributed modulo 1 (abbreviated u.d. mod 1 ) if for every pair $a, b$ of real numbers with $0 \leq a<b \leq 1$ we have $\lim _{N \rightarrow \infty} \frac{A([a, b) ; N ; \omega)}{N}=b-a$. The formal definition of u.d. $\bmod 1$ was given by Weyl [3] [4]. Let $\Delta: 0=z_{0}<z_{1}<z_{2}<\ldots$ be a subdivision of the interval $[0, \infty)$ with $\lim _{k \rightarrow \infty} z_{k}=\infty$. For $z_{k-1} \leq x<z_{k}$ put $[x]_{\Delta}=z_{k-1}$ and $\{x\}_{\Delta}=\frac{x-z_{k-1}}{z_{k}-z_{k-1}}$ so that $0 \leq\{x\}_{\Delta}<1$. The sequence of $\left(x_{n}\right)$, $n=1,2,3, \ldots$ of non-negative real numbers is said to be uniformly distributed modulo $\Delta$ (abbreviated u.d. mod $\Delta$ ) if the sequence $\left(\left\{x_{n}\right\}_{\Delta}\right), n=1,2,3, \ldots$ is u.d. mod 1. The notion of u.d. mod $\Delta$ was introduced by Levaque [5].

If $f$ is a function having a Riemann integral in the interval $[a, b]$, then its integral is the limit of Riemann sums taken by sampling the function $f$ in a set of points chosen from a fine partition of the interval. This is then a criterion for determining if a sequence is uniformly distributed. A sequence of real numbers is uniformly distributed $(\bmod 1)$ if and only if for every Riemann-integrable function $f$ on $[0,1]$ one has $\lim _{N \rightarrow \infty} 1 / N \sum_{n \leq N} f\left(\left\{x_{n}\right\}\right)=\int_{0}^{1} f(x) d x$. In the 
following, evidence that $n-x$ and $x$ are $\mathrm{u} . \mathrm{d}$. $\bmod \Delta$ is presented using this criterion and Weyl's criterion [3] [4].

Weyl's criterion is that $\left(\gamma_{n}\right)$ is u.d. $\bmod 1$ if and only if $\lim _{N \rightarrow \infty} 1 / N \sum_{n=1}^{N} e^{2 \pi i m \gamma_{n}}=$ 0 for every integer $m \neq 0$. In the following, the $z$ increments in the subdivision are set to $\sqrt{2}$ to avoid any aliasing with the integer $n-x$ and $x$ values. A plot of the resulting sequence generated from the sorted $n-x$ values for $n=1,2,3, \ldots, l-1$ and $l=997$ is

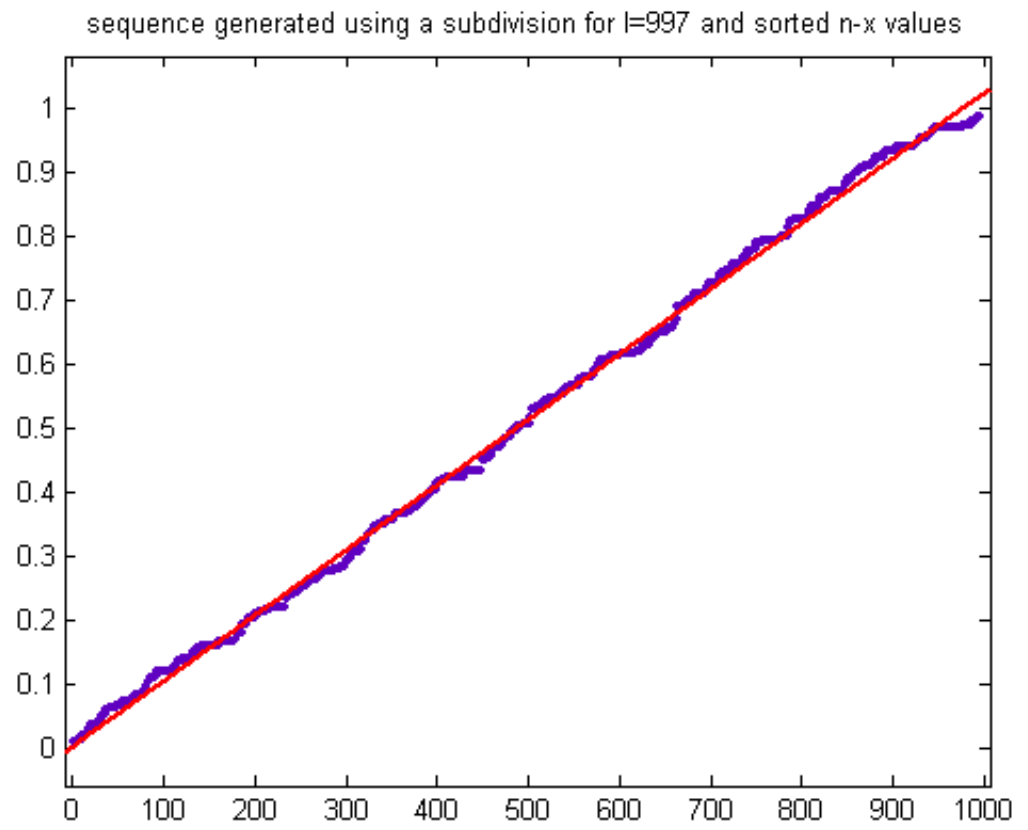

Setting $\left(\gamma_{n}\right)$ to such sequences in Weyl's formula and computing the moduli of the complex-valued results for $l=1999, n-x$, and $m=1$ gives 


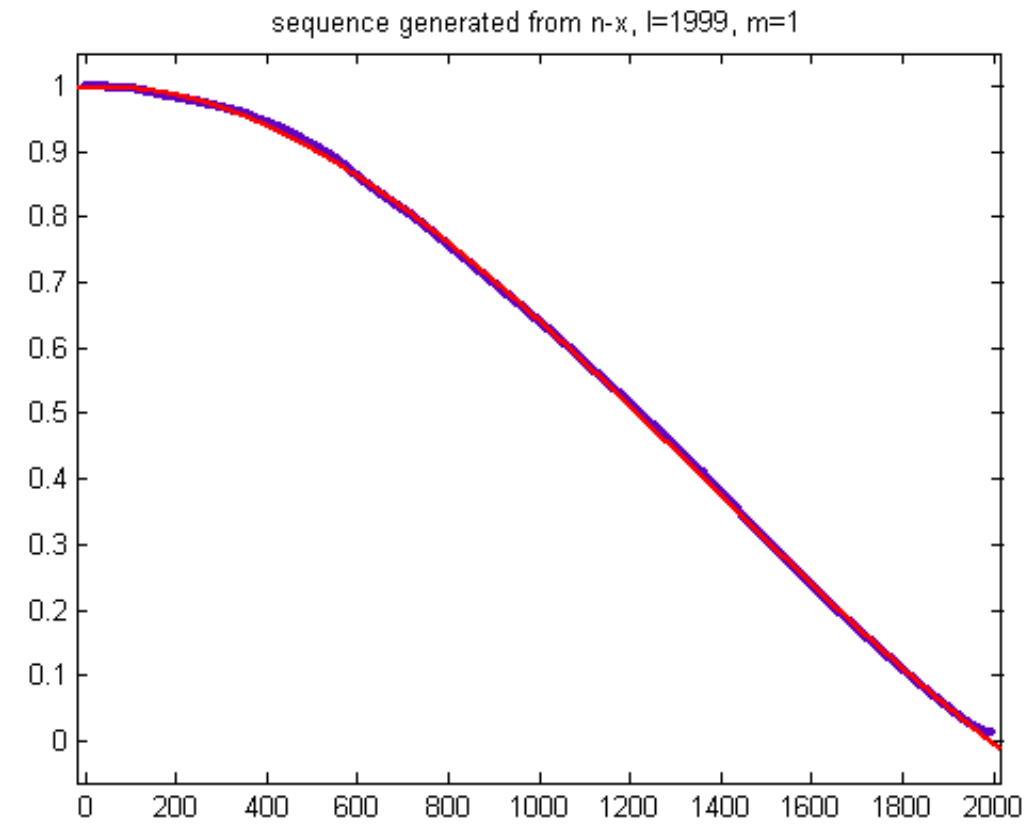

A cubic least-squares fit of the curve (where R-squared=0.9999) is included. The moduli for $l=1999, x$, and $m=1$ (excluding 16 values of zero in the input sequence) are 


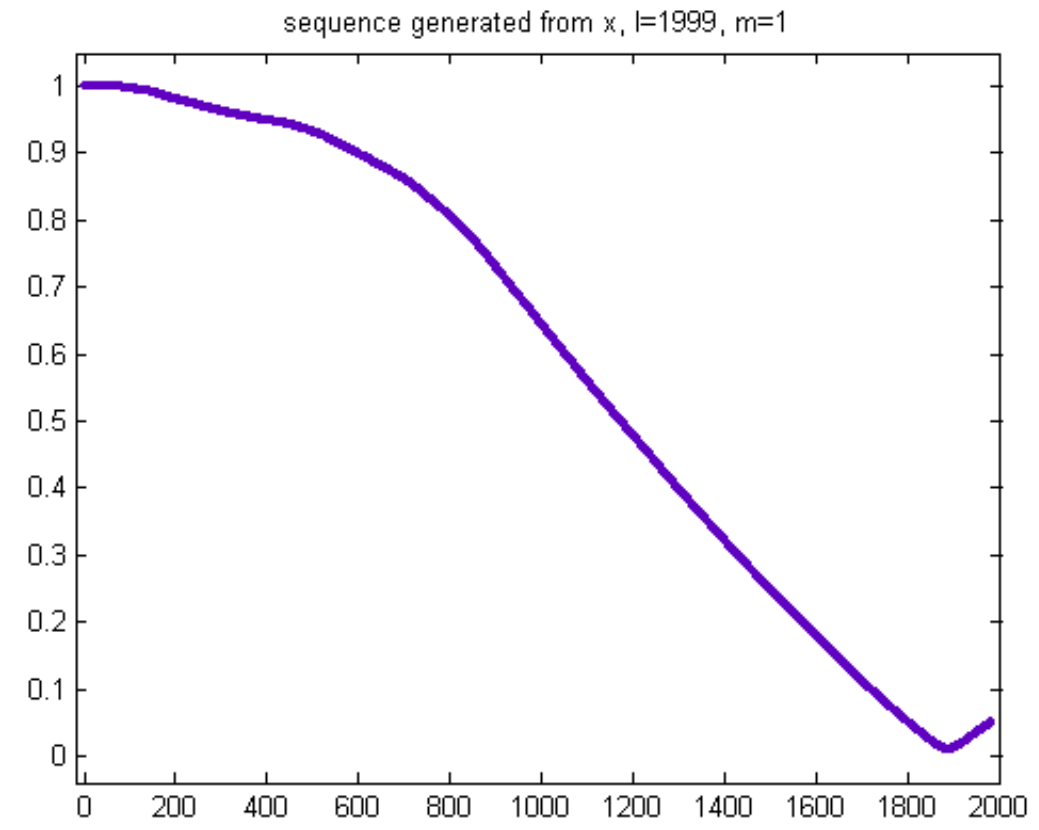

The moduli for $l=3989, n-x$, and $m=2$ are

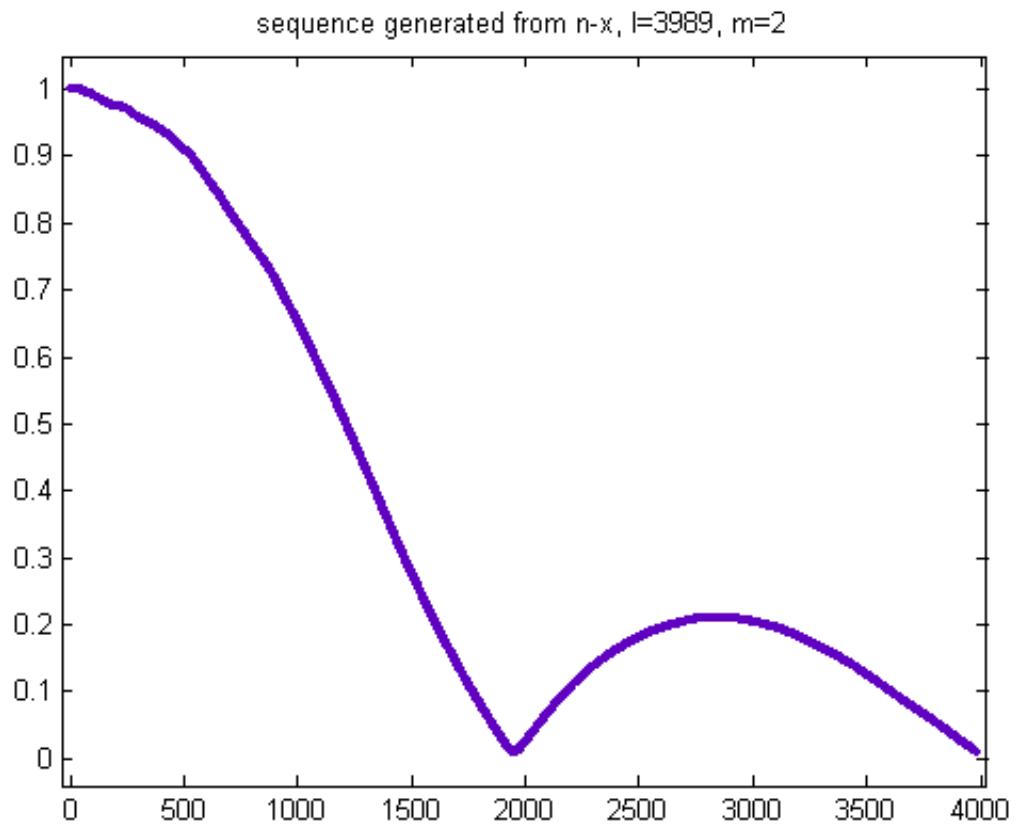


The moduli for $l=3989, x$, and $m=2$ (excluding 6 values of zero in the input sequence) are

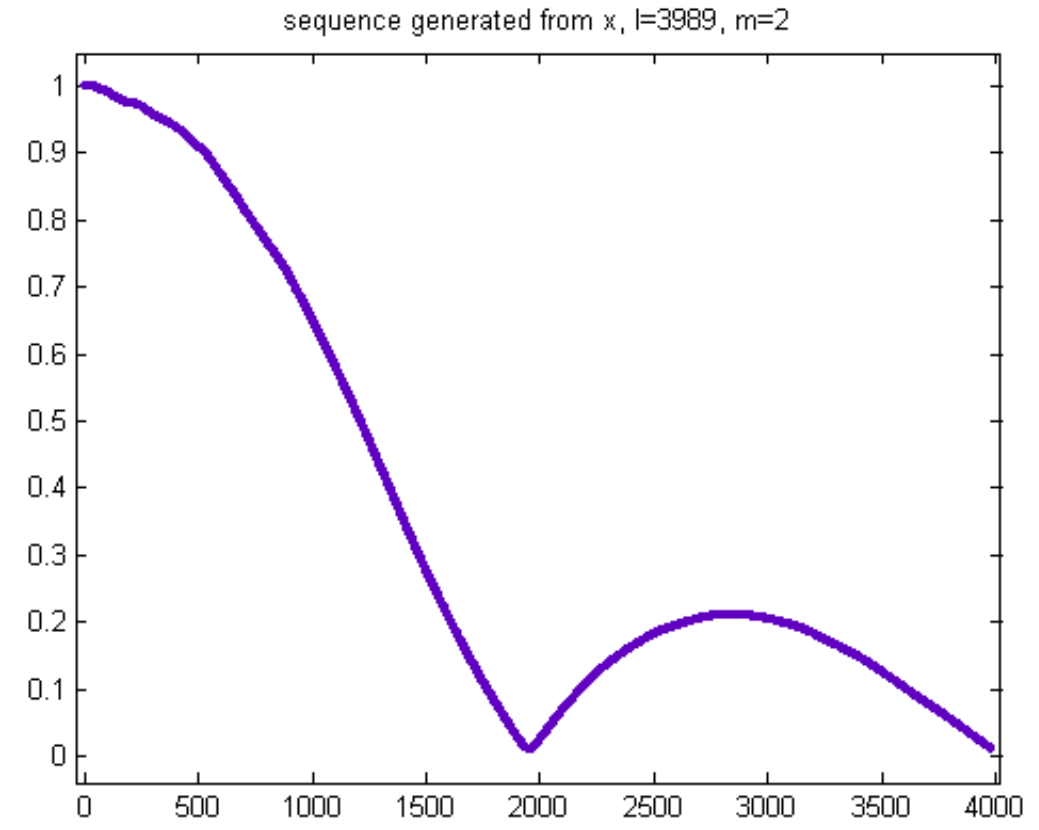

The moduli for $l=5987, n-x$, and $m=3$ are 


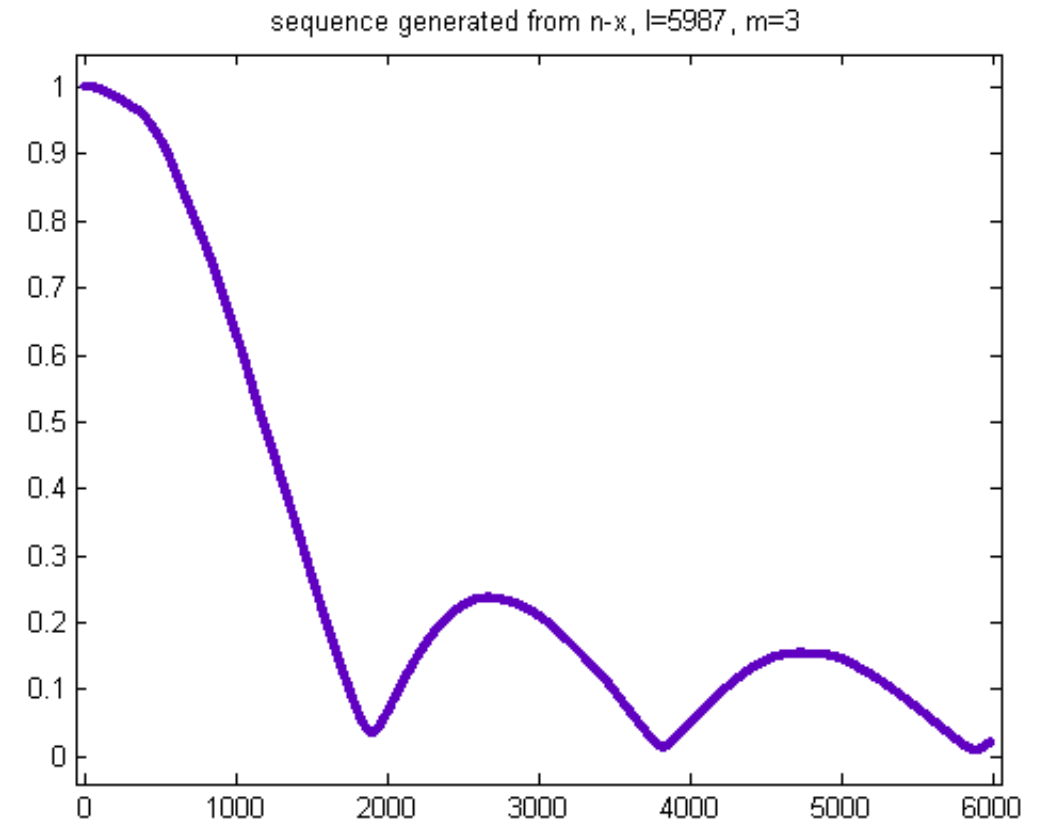

The moduli for $l=5987, x$, and $m=3$ (excluding 8 values of zero in the input sequence) are 


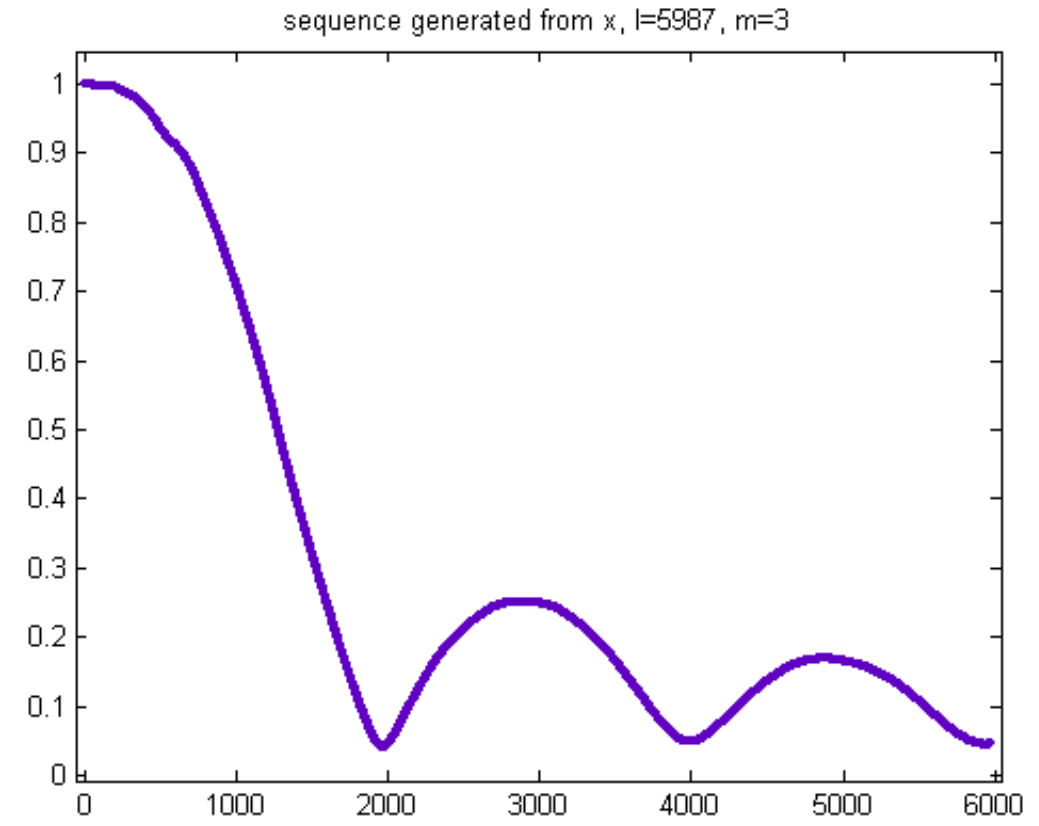

The moduli for $l=7993, n-x$, and $m=4$ are

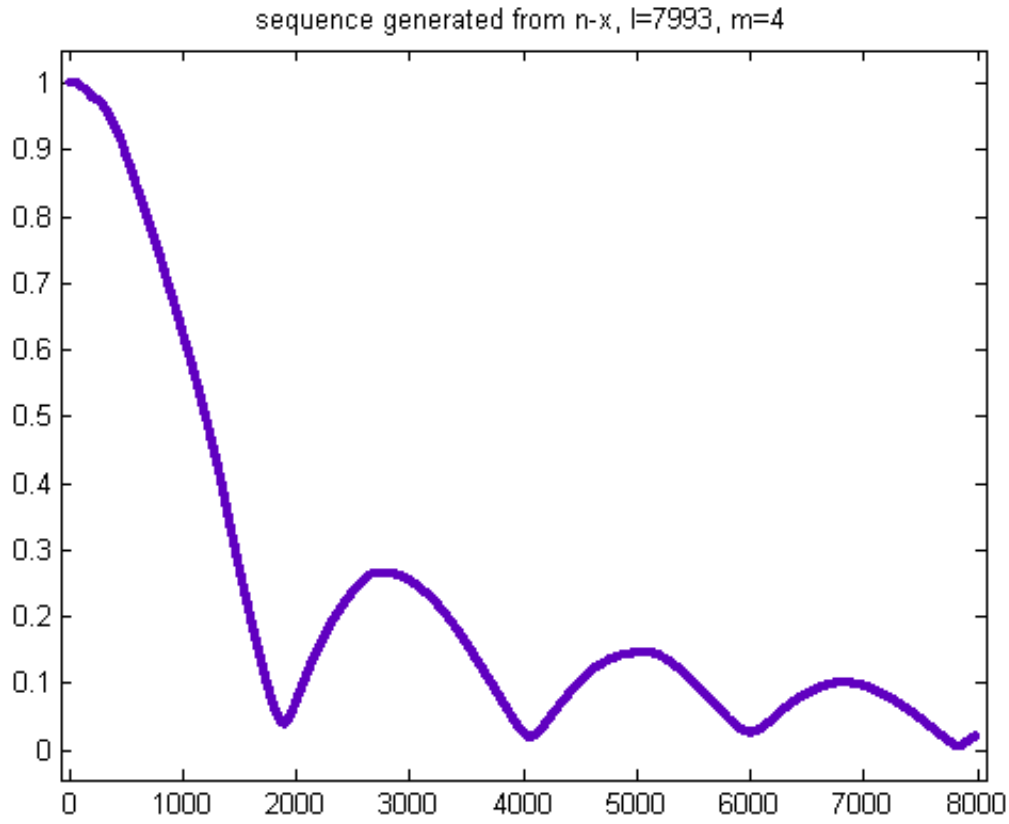


The moduli for $l=7993, x$, and $m=4$ (excluding 32 values of zero in the input sequence) are

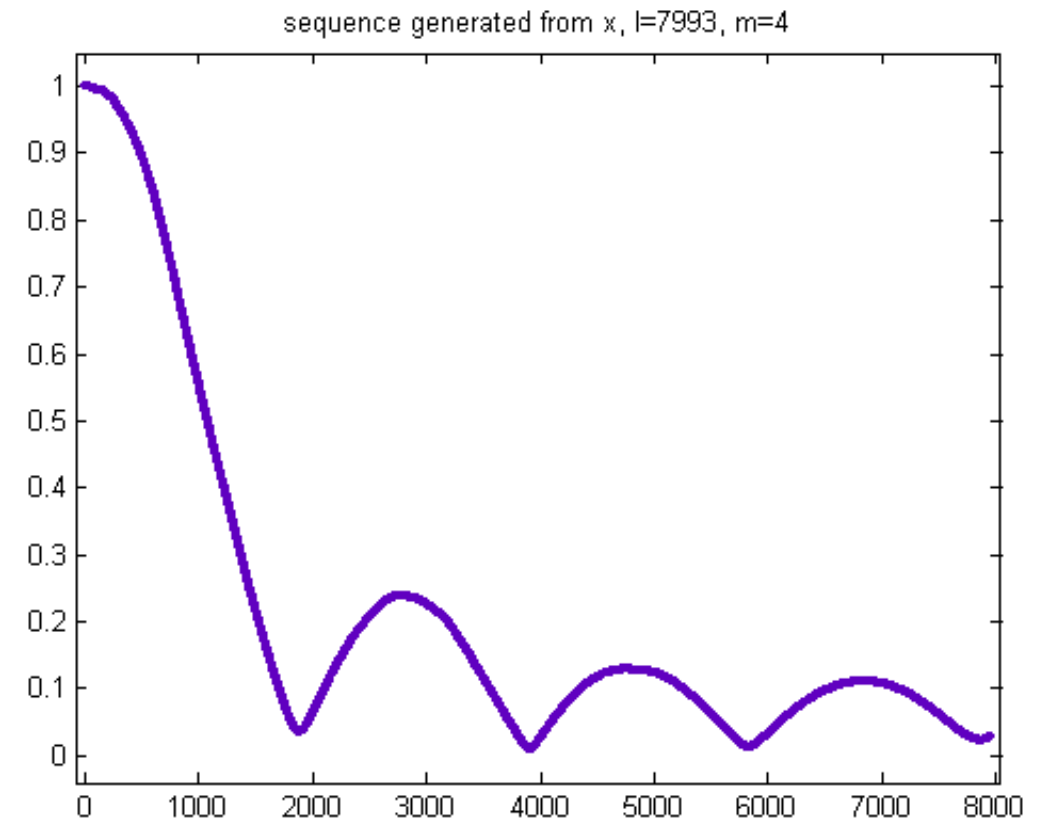

The moduli for $l=9973, n-x$, and $m=10$ are 


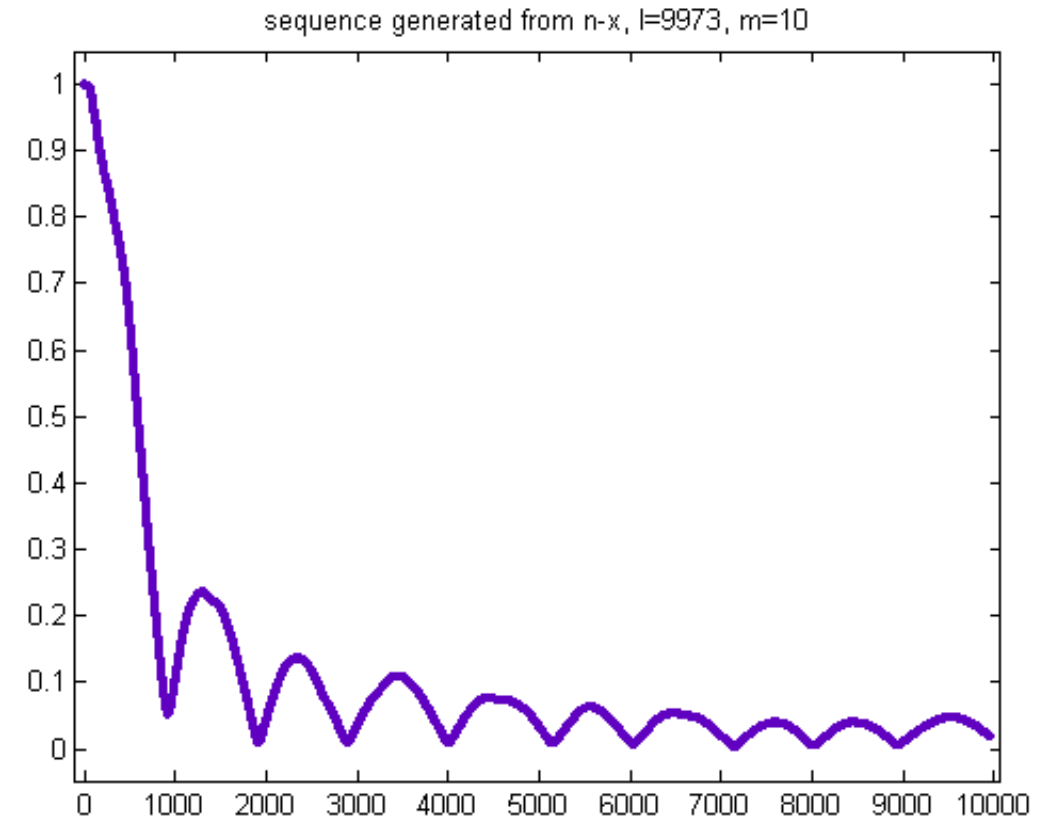

The moduli for $l=9973, x$, and $m=10$ (excluding 18 values of zero in the input sequence) are 


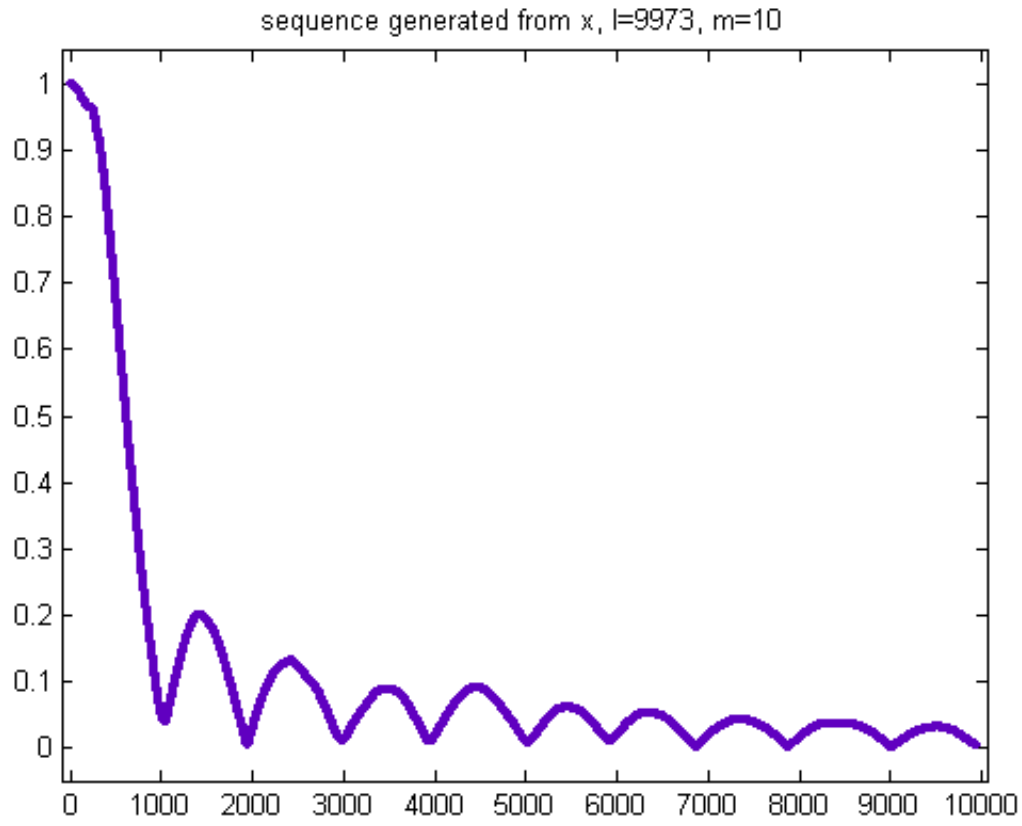

The functions $f(x)$ to be considered are $x, x^{2}, x^{3}, x^{4}, \sqrt{x}, \sqrt{\sqrt{x}}, \log (x), e^{x}$, $\sin (x), \cos (x), \tan (x)$, and $\frac{1}{a^{2}+x^{2}}$. The values of $\int_{0}^{1} f(x) d x$ are $1 / 2,1 / 3,1 / 4$, $1 / 5,2 / 3,4 / 5,-1,2.72,0.84,0.54,1.56$, and $\frac{1}{a} \tan ^{-1} \frac{x}{a}$ (equal to 0.23 for $a=2$ and 0.11 for $a=3$ ) respectively. For $l=997$ and the sequence generated from $x$, the results are $0.49,0.31,0.22,0.17,0.66,0.80,-1.00,2.69,0.84,0.56,1.45,0.23$ (for $a=2$ ), and 0.11 (for $a=3$ ) respectively. For $l=9973$ and the sequence generated from $n-x$, the results are $0.50,0.34,0.25,0.20,0.67,0.80,-0.97$, $2.74, .84, .54,1.55,0.23$ (for $a=2$ ), and 0.11 (for $a=3$ ) respectively.

A plot of the integral for $f(x)=x^{2}$ is 


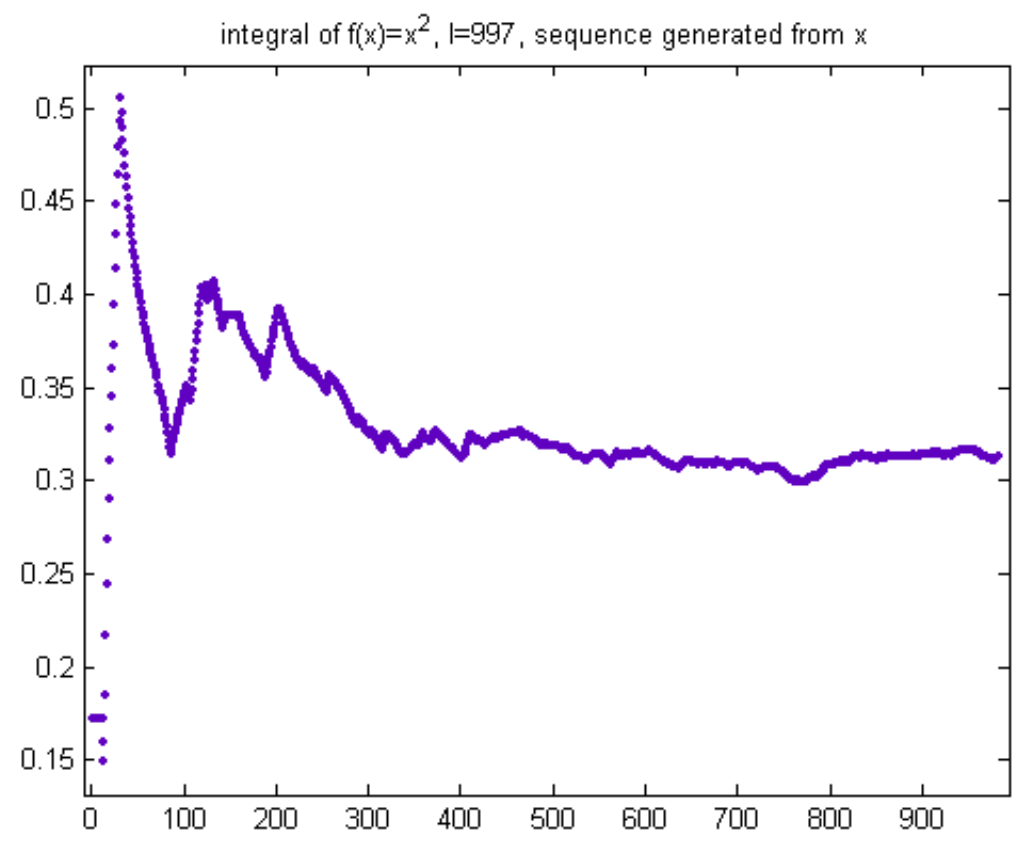

A plot of the integral for $f(x)=e^{x}$ is

integral of $f(x)=e^{x}, 1=997$, sequence generated from $x$

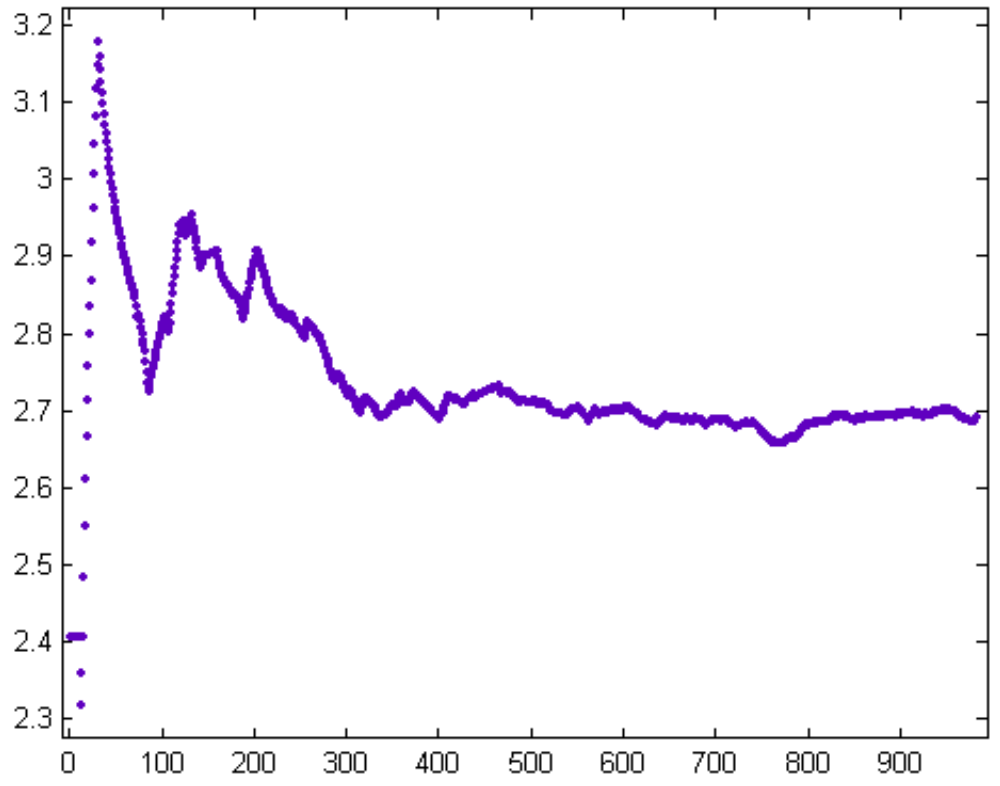


A plot of the integral for $f(x)=\sin (x)$ is

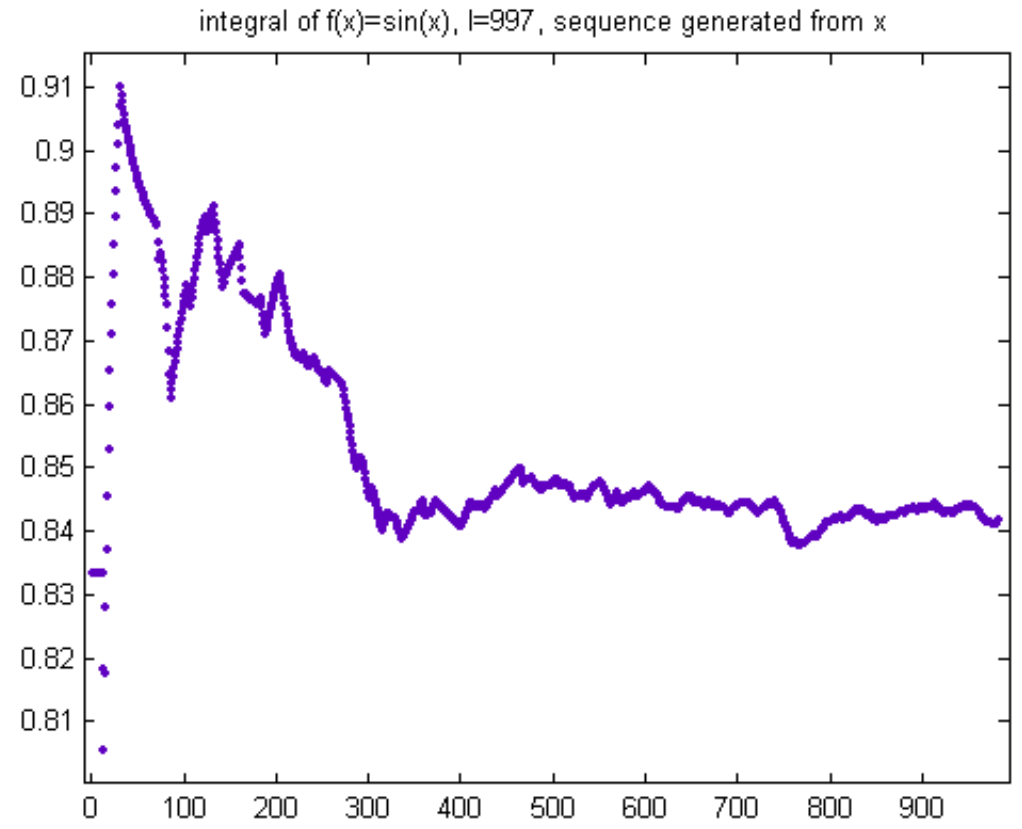

A plot of the integral for $f(x)=\frac{1}{a^{2}+x^{2}}, a=3$ is 


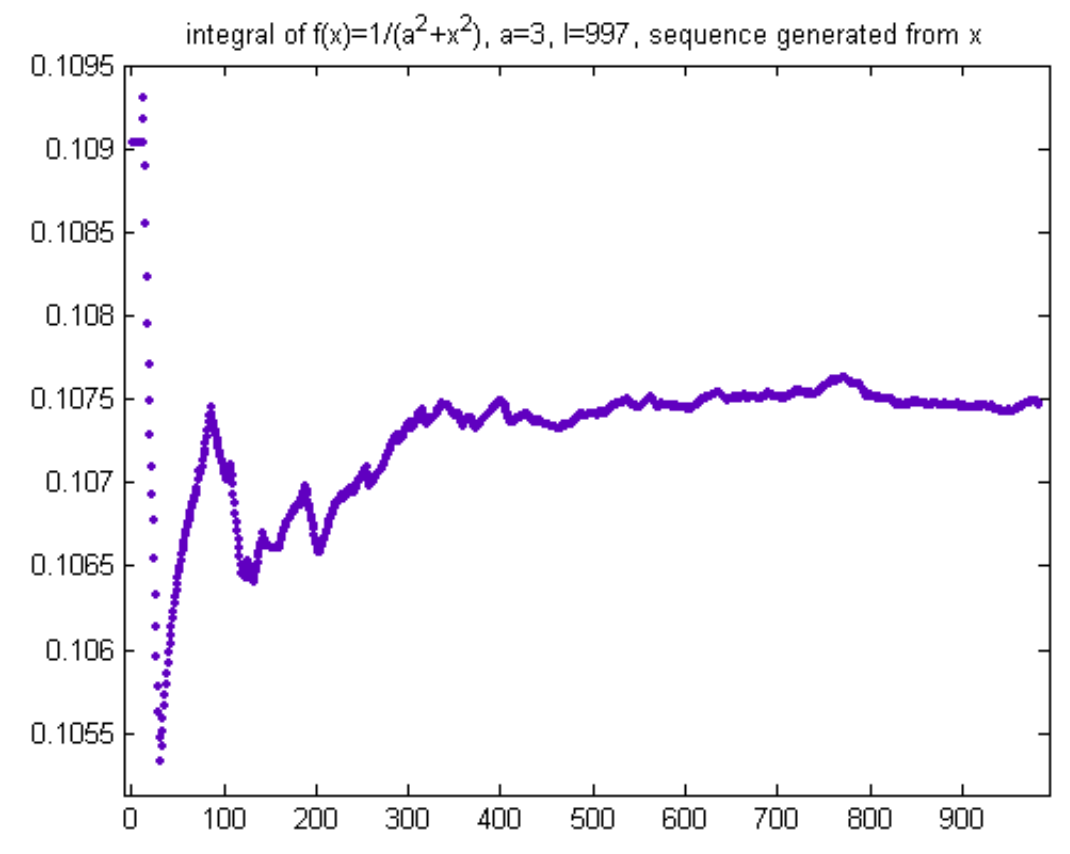

The trigonometric functions require a fixed amount to be added to the sequence values (apparently to change the phase). The exponential function also requires a fixed amount to be added to the sequence values - the same as for the cosine function. Apparently, this is due to Euler's formula $e^{i x}=$ $\cos (x)+i \cdot \sin (x)$. Denote the amounts for sine and cosine by $j$ and $k$ respectively. These values satisfy the equation $j^{2}+k^{2}=\cos (1)$, similar to the formula $\sin (x)^{2}+\cos (x)^{2}=1$. They also satisfy the equation $j / k=\sqrt{\tan (1)}$, similar to the formula $\sin (x) / \cos (x)=\tan (x)$. The amount required for the sine function is $\sin ^{-1}(\cos (1))$. The amount required for the cosine function can be determined by using the formula $j^{2}+k^{2}=\cos (1)$. The amount required for the tangent function is $1 / e(e=\tan (1) / j)$.

\section{References}

[1] Cox, Darrell Ghosh, Sourangshu Sultanow, Eldar. (2021). Collatz Cycles and 3n + c Cycles. 10.13140/RG.2.2.26544.38400.

[2] D.Cox, S. Ghosh and E.Sultanow, Collatz Cycles and 3n+c Cycles, arXiv:2101.04067 [math.GM] (2021)

[3] Weyl, H., Über ein Problem aus dem Gebiete der diophantischen Approximationen, Nachr. Ges. Wiss. Göttingen, Math.-phys. Kl., 1914, 234-244

[4] Weyl, H., Über die Gleichverteilung von Zahlen mod. Eins, Math. Ann., 77, 313-352 (1916) 
[5] LeVeque, W.J., On uniform distribution modulo a subdivision, Pacific J. Math.,3, 757-771 (1953) 